\title{
Influence Of Rotation, Variable Viscosity And Temperature On Peristaltic Transport In An Asymmetric Channel
}

\author{
${ }^{1}$ Alaa Waleed Salih and ${ }^{2}$ Sundos Bader Habeeb \\ 1,2 Department of Mathematics ,College of Science, University of Baghdad, Baghdad ,Iraq. \\ Email:alaa.w@sc.uobaghdad.edu.iq. \\ (ORCID: 0000-0001-8438-0108)
}

Article History: Received: 11 January 2021; Accepted: 27 February 2021; Published online: 5 April 2021

\begin{abstract}
In this paper we investigates the effects of each rotation, variable viscosity and temperature on the peristaltic phenomena in an asymmetric channel. The motion and heat equations are obtained in Cartesian coordinates, the dimensionless form of the governing equation are controlled by many dimensionless number e.g. Reynolds, Hartmann, Grashof, Prandle ...These equations are nonlinear and to simplify ,the long wave length and low Reynolds number is used. The resulting dimensionless equation are then solved analytically by using perturbation expansion about Reynold model viscosity number. The effects of different parameter on axial velocity, stream function, pressure rise and heat distribution are analysis graphically by using the mathematica package.
\end{abstract}

Keywords: Peristaltic flow ,Heat transfer, variable viscosity, porous medium, rotation.

\section{Introduction.}

Nowadays the peristaltic transport of physiological fluids appeared by progressive wave $\mathrm{f}$ area contraction or expansion along the length of flexible tube. it is involved in biological systems such as swallowing food through the esophagus, motion of chime in gastro-intestinal tract . also, this transport work on the same of peristaltic pump are used for industrial and medical application. The physical mechanism of the flow induced by the traveling wave can be well understood and is known as the peristaltic transport mechanism .

Many researchers study the Peristaltic phenomenon in different subject and its application .Most of these studies were applied under constant viscosity[1,2] .several recent $[3,5]$ investigated the impact of variable viscosity , when the viscosity is dependent on the distance only. Elshehawey and Gharsseldien [6] studied the effects of variable viscosity for peristaltic motion of an incompressible Newtonian fluid through a channel with three layers flow. However some researchers are studied the influence of heat and rotation in peristaltic flow .Hayat et al.[7] investigated the peristaltic motion of MHD with non-Newtonian fluid in an inclined asymmetric channel and low Reynolds number. In recent years, considerable efforts have been usefully devoted to the study of peristaltic flow of non-Newtonian fluids because a practical and fundamental relation that can be used for all fluids and flow is not available. Mahmoud S R et al [8] discussed the influence of the rotation on the wave motion through a cylindrical bore in a micropolar porous medium .Siddiqui and Schehaawey [9] analyze the mechanics of peristaltic pumping of non-Newtonian fluid through an axisymmetric conduit . The extensive literature on the topic is now available and we can mention a few recent interesting in Refs.[10-13] . Abd-Alla et al.[14] concerned with rotation effect on peristaltic transport in an asymmetric channel of Jeffery fluid .the extensive literature on the topic is now available and we can mention afew recent in Ref. [15-16]

The study of heat transfer analysis is an important area in connection with peristaltic motion, which has application such as sanitary fluid transport, blood pumps in heart lungs machine and corrosive transport of fluids where the contact of fluid with machinery parts is prohibited.

The aim of this paper was to study the impact of rotation and variable viscosity with heat transfer on the peristaltic transport of Non -Newtonian fluid in asymmetric channel . The influence of various pertinent parameters on the flow characteristics, Where study are discussed through graphs.

\section{Formulation of the Problem}

Consider the unsteady incompressible peristaltic transport of second -order fluid through a porous medium and an asymmetric two dimensional channel of width $d_{1}+d_{2}$ The lower wall of the channel is maintained at temperature $_{1}$ while the upper wall has temperature $\mathrm{T}_{0}$ as shown in the Fig .1 . 


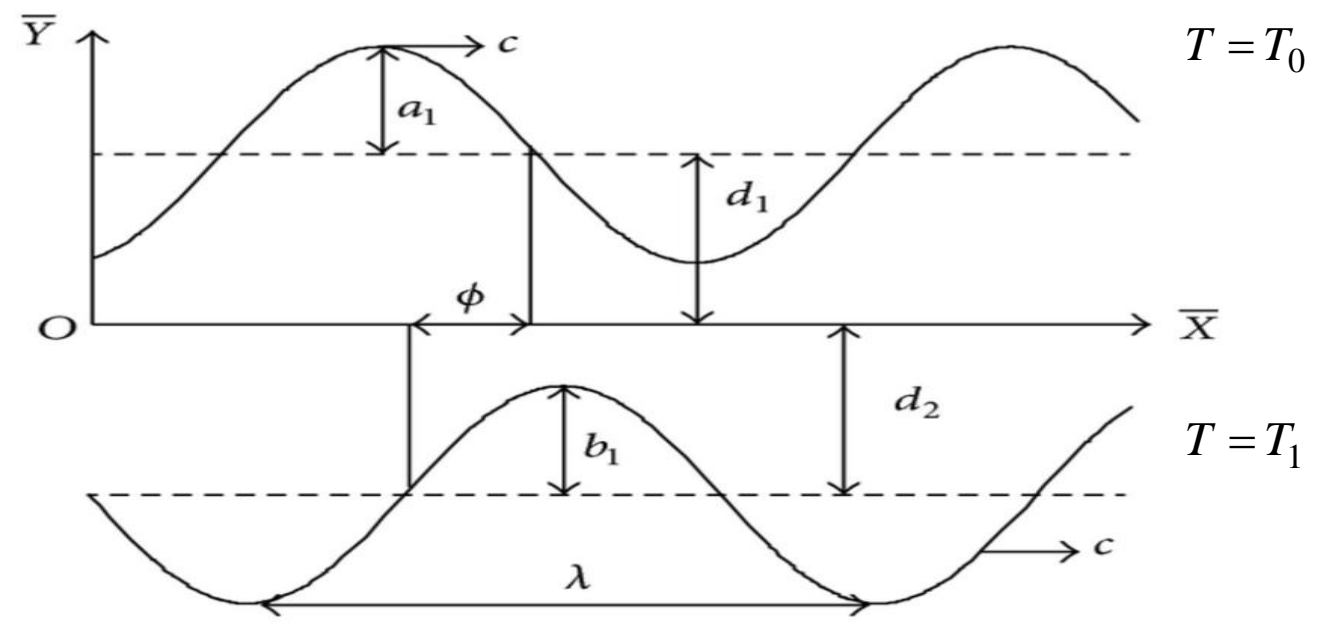

Fig.1 : Schematic diagram of a two - dimensional asymmetric channel

The geometry of the wall surface is define as :

, upper wall

(1)

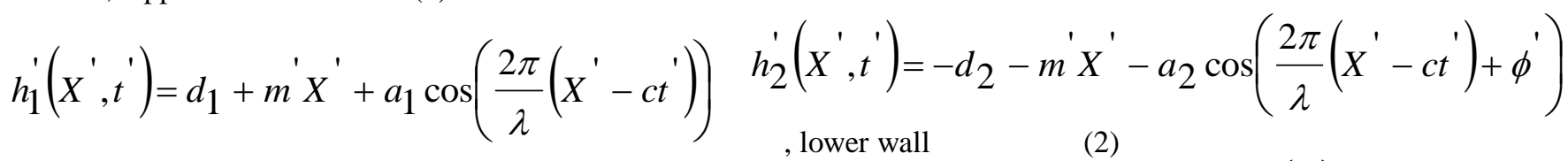

which $\left(a_{1}\right)$ and $\left(a_{2}\right)$ are amplitudes of the waves, $(\lambda)$ is the wavelength, $(c)$ is the wave speed, $\left(t^{\prime}\right)$ is the time $\phi(0 \leq \phi \leq \pi)$ is phase difference and $\left(m^{\prime} \ll 1\right)$ is a non-uniform parameter.$\left(X^{\prime}, Y^{\prime}\right)$ are the Cartesian coordinates, where $X$ ' is the direction of wave propagation while $Y$ ' is taken normal to it . $\phi=0$ corresponds to symmetric channel with waves out of phase and $\phi=\pi$ the waves are in phase.

\section{The Governing Equations}

The governing equations of motion of MHD fluid model, with variable viscosity, through an asymmetric channel in laboratory frame are

The continuity equation

$\frac{\partial U^{\prime}}{\partial X^{\prime}}+\frac{\partial V^{\prime}}{\partial Y^{\prime}}=0$

The momentum equations

$$
\begin{aligned}
& \rho\left(U^{\prime} \frac{\partial U^{\prime}}{\partial X^{\prime}}+V^{\prime} \frac{\partial U^{\prime}}{\partial Y^{\prime}}\right)-\rho \Omega\left(\Omega U^{\prime}+2 \frac{\partial V^{\prime}}{\partial t^{\prime}}\right)=-\frac{\partial P^{\prime}}{\partial X^{\prime}}+2 \frac{\partial}{\partial X^{\prime}}\left(\mu^{\prime}\left(Y^{\prime}\right) \frac{\partial U^{\prime}}{\partial X^{\prime}}\right) \\
& +\frac{\partial}{\partial Y^{\prime}}\left(\mu^{\prime}\left(Y^{\prime}\right) \frac{\partial V^{\prime}}{\partial X^{\prime}}+\frac{\partial U^{\prime}}{\partial Y^{\prime}}\right)-\sigma B_{0}^{2} U^{\prime}-\frac{\mu^{\prime}\left(Y^{\prime}\right)}{k}\left(U^{\prime}-c\right) \\
& \rho\left(U^{\prime} \frac{\partial V^{\prime}}{\partial X^{\prime}}+V^{\prime} \frac{\partial V^{\prime}}{\partial Y^{\prime}}\right)-\rho \Omega\left(\Omega V^{\prime}+2 \frac{\partial U^{\prime}}{\partial t^{\prime}}\right)=-\frac{\partial P^{\prime}}{\partial Y^{\prime}}+2 \frac{\partial}{\partial Y^{\prime}}\left(\mu^{\prime}\left(Y^{\prime}\right) \frac{\partial V^{\prime}}{\partial Y^{\prime}}\right) \\
& \left.+\frac{\partial}{\partial X^{\prime}}\left(\mu^{\prime}\left(Y^{\prime}\right) \frac{\partial V^{\prime}}{\partial X^{\prime}}+\frac{\partial U^{\prime}}{\partial Y^{\prime}}\right)-\frac{\mu^{\prime}\left(Y^{\prime}\right)}{k} V^{\prime}\right)
\end{aligned}
$$


The energy equation

$$
\rho C_{p}\left(\frac{\partial T^{\prime}}{\partial t^{\prime}}+U^{\prime} \frac{\partial T^{\prime}}{\partial X^{\prime}}+V^{\prime} \frac{\partial T^{\prime}}{\partial Y^{\prime}}\right)=k\left(\frac{\partial^{2} T^{\prime}}{\partial X^{\prime}}+\frac{\partial^{2} T^{\prime}}{\partial Y^{\prime}}\right)+\phi_{0}
$$

In the laboratory frame $\left(X^{\prime}, Y^{\prime}\right)$ the flow is unsteady. if observed a coordinate system moving at wave speed c in the wave frame $\left(x^{\prime}, y^{\prime}\right)$, it can be noted as steady.

In the two frames, the coordinate, velocities, pressure, and temperature are

$$
x^{\prime}=X^{\prime}-c t^{\prime}, y^{\prime}=Y^{\prime}, u^{\prime}=U^{\prime}-c, v^{\prime}=V^{\prime}, p^{\prime}=P^{\prime}, T=T^{\prime} \text {, }
$$

In order to simplify the governing equations of the motion , We introducing the following non-dimensional parameters :

$$
\begin{aligned}
& x=\frac{x}{\lambda}, y=\frac{y^{\prime}}{d^{\prime}}, u=\frac{u^{\prime}}{c}, v=\frac{\lambda v^{\prime}}{d_{1} c}, p=\frac{d_{1}^{2} p^{\prime}\left(x^{\prime}\right)}{c \mu \lambda}, t=\frac{c t^{\prime}}{\lambda}, \\
& h_{1}(x)=\frac{h_{1}^{\prime}\left(x^{\prime}\right)}{d_{1}}, h_{2}(x)=\frac{h_{2}^{\prime}\left(x^{\prime}\right)}{d_{1}}, \mu(y)=\frac{\mu^{\prime}\left(y^{\prime}\right)}{\mu_{0}}, \\
& \delta=\frac{d_{1}}{\lambda}, \theta=\frac{T-T_{0}}{T_{1}-T_{0}},
\end{aligned}
$$

Where $x, y, u, v, t, p, \theta, \delta, \mu_{0}$ are the components of the dimensionless coordinates, the dimensionless axial velocity, the dimensionless time, the dimensionless pressure, the dimensionless temperature, the wave number, the constant viscosity respectively. $\Delta T=\left(T_{1}-T 0\right)$ denote the temperature difference .

By using the dimensionless quantities in (7), the equations (3) to (6) become

$$
\frac{\partial u}{\partial x}+\frac{\partial v}{\partial y}=0
$$

$$
\begin{aligned}
& R_{e} \delta\left((u+1) \frac{\partial u}{\partial x}+v \frac{\partial u}{\partial y}\right)-\frac{\rho \Omega^{2} d_{1}^{2}}{\mu_{0}} u-2 \Omega R_{e} \delta^{2} \frac{\partial v}{\partial t}=-\frac{\partial p}{\partial x}+2 \delta \frac{\partial}{\partial x}\left(\mu(y) \frac{\partial u}{\partial x}\right)+ \\
& \frac{\partial}{\partial y}\left(\mu(y)\left(\delta^{2} \frac{\partial v}{\partial x}+\frac{\partial u}{\partial y}\right)\right)-M^{2}(u+1)-D^{2}(u+1)+G_{r} \theta \\
& R_{e} \delta^{3}\left((u+1) \frac{\partial v}{\partial x}+v \frac{\partial v}{\partial y}\right)-\frac{\rho \Omega^{2} d_{1}^{2}}{\mu_{0}} \delta^{2} v-2 \Omega R_{e} \delta^{2} \frac{\partial u}{\partial t}=-\frac{\partial p}{\partial y}+2 \delta^{2} \frac{\partial}{\partial y}\left(\mu(y) \frac{\partial v}{\partial y}\right)+ \\
& \frac{\partial}{\partial x}\left(\mu(y)\left(\delta^{4} \frac{\partial v}{\partial x}+\delta^{2} \frac{\partial u}{\partial y}\right)\right)-D \delta^{2} v \\
& R_{e} \delta P_{r}\left((u+1) \frac{\partial \theta}{\partial x}+v \frac{\partial \theta}{\partial y}\right)=\left(\delta^{2} \frac{\partial^{2} \theta}{\partial x^{2}}+\frac{\partial^{2} \theta}{\partial y^{2}}\right)+\beta
\end{aligned}
$$


Where $R_{e}, M, G_{r}, \beta, P_{r}, D$ are non- dimensional parameters, called respectively the Reynolds number ,Hartmann number, Grashof number, source/sink parameter and Prandtl number given by

$$
\begin{aligned}
& R_{e}=\frac{\rho c d_{1}}{\mu_{0}} \quad, \quad M=\sqrt{\frac{\delta}{\mu_{0}}} B_{0}^{2} d_{1}^{2}, G_{r}=\frac{\rho g \alpha\left(T_{1}-T_{0}\right) d_{1}^{2}}{\mu_{0} c}, \\
& P_{r}=\frac{\mu_{0} c p}{k} \quad, \quad \beta=\frac{\phi_{0} d_{1}^{2}}{k\left(T_{1}-T_{0}\right)} \quad, \quad D=\frac{d_{1}^{2}}{k}
\end{aligned}
$$

Introducing the stream function $\psi(x, y, t)$ that relationship with velocity components defined by

$$
u=\frac{\partial \psi}{\partial y}, \quad v=-\frac{\partial \psi}{\partial x},
$$

under the assumptions of long wavelength approximation $(\delta \ll 1)$ and low Reynolds number $\left(R_{e} \rightarrow 0\right)$, the Eqs. (8-11) will take the form

$$
\begin{aligned}
& -\frac{\rho \Omega^{2} d_{1}^{2}}{\mu_{0}} \frac{\partial \psi}{\partial y}=-\frac{\partial p}{\partial x}+\frac{\partial}{\partial y}\left(\mu(y) \frac{\partial^{2} \psi}{\partial y^{2}}\right)-M^{2}\left(\frac{\partial \psi}{\partial y}+1\right)-D\left(\frac{\partial \psi}{\partial y}+1\right)+G_{r} \theta \\
& 0=-\frac{\partial p}{\partial y} \\
& 0=\frac{\partial^{2} \theta}{\partial y^{2}}+\beta
\end{aligned}
$$

The above problem, will be solve subject to the following suitable boundary conditions :

$$
\begin{array}{ll}
\psi=\frac{F}{2}, \frac{\partial \psi}{\partial y}+\beta_{1} \frac{\partial^{2} \psi}{\partial y^{2}}=-1 & \text { at } y=h_{1} \\
\theta+\gamma \frac{\partial \theta}{\partial y}=0 & \text { at } y=h_{1} \\
\psi=-\frac{F}{2}, \frac{\partial \psi}{\partial y}-\beta_{1} \frac{\partial^{2} \psi}{\partial y^{2}}=-1 & \text { at } y=h_{2} \\
\theta-\gamma \frac{\partial \theta}{\partial y}=0 & \text { at } y=h_{2}
\end{array}
$$

Although the viscosity $\mu$ depends on $y$ and $\theta$,we consider the following form was proposed by Slattery [17].

$$
\mu(y)=e^{-\alpha y} \quad \text { or } \quad \mu(y)=1-\alpha y \quad \text { for } \quad \alpha \ll 1 .
$$

Where $\alpha$ is Reynolds model viscosity parameter that we considered to be very small. The axial pressure can be written in the form

$$
\frac{d p}{d x}=(1-\alpha y) \frac{\partial^{3} \psi}{\partial y^{3}}-\alpha \frac{\partial^{2} \psi}{\partial y^{2}}+\left(\frac{\rho \Omega^{2} d_{1}^{2}}{\mu_{0}}-N^{2}\right) \frac{\partial \psi}{\partial y}-N^{2}+G_{r} \theta
$$

The solution of temperature on Eq. ( 16 ) subject to the associated boundary conditions ( 17-20) yields ..

$$
\theta=-\frac{\beta y^{2}}{2}+c_{1} y+c_{2} \text {, }
$$


Where the integrating constant $c_{i}, i=1,2$ obtained using boundary conditions ( 17)\& (18), by the following

$$
\begin{aligned}
& c_{1}=\frac{1}{2}\left(-h_{1} h_{2} \beta+\left(h_{1}-h_{2}\right) \beta\right), \\
& c_{2}=\frac{1}{2} \beta\left(h_{1}+h_{2}\right)
\end{aligned}
$$

We can widen the stream function $(\psi)$, pressure $(P)$, and the flow rate $(F)$ in a power series form, as follows

$$
\begin{aligned}
& \psi=\psi_{0}+\alpha \psi_{1}+\alpha^{2} \psi_{2}+\ldots \\
& P=P_{0}+\alpha P_{1}+\alpha^{2} P_{2}+\ldots \\
& F=F_{0}+\alpha F_{1}+\alpha^{2} F_{2}+\ldots
\end{aligned}
$$

Then obtains the following two system zeroth-order and first - order equations :

$$
\frac{\partial^{4} \psi_{0}}{\partial y^{4}}-S^{2} \frac{\partial^{2} \psi_{0}}{\partial y^{2}}+G_{r} \frac{\partial \theta}{\partial y}=0
$$

With the corresponding boundary conditions

$$
\begin{array}{ll}
\psi_{0}=\frac{F_{0}}{2} \text { and } \frac{\partial \psi_{0}}{\partial y}+\beta_{1} \frac{\partial^{2} \psi_{0}}{\partial y^{2}}=-1 & \text { at } y=h_{1} \\
\psi_{0}=-\frac{F_{0}}{2} \text { and } \frac{\partial \psi_{0}}{\partial y}-\beta_{1} \frac{\partial^{2} \psi_{0}}{\partial y^{2}}=-1 & \text { at } y=h_{2}
\end{array}
$$

And ,the first-order equation is found in the form

$$
\frac{\partial^{4} \psi_{1}}{\partial y^{4}}-y \frac{\partial^{4} \psi_{0}}{\partial y^{4}}-2 \frac{\partial^{3} \psi_{0}}{\partial y^{3}}-S^{2} \frac{\partial^{2} \psi_{1}}{\partial y^{2}}=0
$$

The corresponding boundary conditions are

$$
\begin{array}{ll}
\psi_{1}=\frac{F_{1}}{2} \quad \text { and } \frac{\partial \psi_{1}}{\partial y}+\beta_{1} \frac{\partial^{2} \psi_{1}}{\partial y^{2}}=0 & \text { at } y=h_{1} \\
\psi_{1}=-\frac{F_{1}}{2} \quad \text { and } \quad \frac{\partial \psi_{1}}{\partial y}-\beta_{1} \frac{\partial^{2} \psi_{1}}{\partial y^{2}}=0 & \text { at } y=h_{2}
\end{array}
$$

Where $S^{2}=N^{2}-\frac{\rho \Omega^{2} d_{1}^{2}}{\mu}, N^{2}=M^{2}+D^{2}$

By using the boundary conditions ( 27) and (28), we can solve Eq.(26), then analytical expression for $\psi_{0}$ ( the final solution for the zeroth order ) may be written as following form ..

$$
\psi_{0}=\frac{c_{2} G_{r} y^{2}-G_{r} y^{3} \beta+2 e^{s y} c_{3}+2 e^{-s y} c_{4}}{6 S^{2}}+c_{5}+y c_{6}
$$

The first order solution gained from the above system is 


$$
\begin{aligned}
& \psi_{1}=\frac{1}{8 S^{4}}\left(-8 c_{2} G_{r} y^{2}+\frac{8}{3} G_{r} y^{3} \beta+e^{s y}\left(-20 c_{3}+17 c_{3} s-\frac{2\left(-4 c_{3} s^{2}+5 c_{3} s^{3}\right) y}{s}+\right.\right. \\
& \left.2 c_{3} s^{3} y^{2}+8 s^{2} c_{7}\right)+e^{-s y}\left(-20 c_{4}-17 c_{4} s-\frac{2\left(4 c_{4} s^{2}+5 c_{4} s^{3}\right) y}{s}-\right.
\end{aligned}
$$

$$
\left.\left.2 c_{4} s^{3} y^{2}+8 s^{2} c_{8}\right)\right)+c_{9}+y c_{10}
$$

The values of coefficients $\left(c_{3}, c_{4}, c_{5}, c_{6}, c_{7}, c_{8}, c_{9}, c_{10}\right)$ are large non - constant and their values can calculated with the boundary conditions by using Mathematica 11. That, also changing when use no-slip conditions in Eqs.(27)\&(30).

The formation of an internally circulating bolus of the fluid by closed streamline is called trapping and this trapped bolus pulled ahead along with the peristaltic wave, the bolus moves with the same speed as that of the wave .

Stream function can be obtained by solved in Mathematica 11 program in form ..,

$$
\begin{aligned}
& \psi=\frac{c_{2} G_{r} y^{2}-G_{r} y^{3} \beta+2 e^{q y} c_{3}+2 e^{-q y} c_{3}}{6 q^{2}}+c_{5}+y c_{6}+\alpha\left(\frac { 1 } { 8 q ^ { 4 } } \left(-8 c_{2} G_{r} y^{2}+\frac{8}{3} G_{r} y^{3} \beta+\right.\right. \\
& e^{q y}\left(-20 c_{3}+17 c_{3} q-\frac{2 y\left(-4 c_{3} q^{2}+5 c_{3} q^{3}\right)}{s}+2 c_{3} q^{3} y^{2}+8 q^{2} c_{7}\right)+ \\
& \left.\left.e^{-q y}\left(-20 c_{4}-17 c_{4} q-\frac{2 y\left(4 c_{4} q^{2}+5 c_{4} q^{3}\right)}{s}-2 c_{4} q^{3} y^{2}+8 q^{2} c_{8}\right)\right)+c_{9}+y c_{10}\right)
\end{aligned}
$$

\section{Numerical Results and Discussion :}

In this section, the results are discussed through the graphical illustrations for different physical quantities . Mathematica program was used to obtain result . Also the trapping phenomenon was studying for the slip condition through graph .

Figs.2-7 show the temperature field for different value of $d, \beta, \gamma, \phi, x, a$, it is noticed the increase in $d, a$ and $\gamma$ that temperature is field decreases while the increase in $\beta$ and $x$, the temperature field increase Thereby the temperature of boundary layer enhanced by appreciable extend .

The variation are shown in Figs. (8-10), respectively, the value of velocity (u) with respect to y axis for different values of physical parameters such as Hartman number $(M)$, Grashof number $\left(G_{r}\right)$.axial velocity increases with increasing Hartman number, whereas is observed in the central region of the artery walls .

Figs. 11 and 12 give some characteristic pressure gradient profiles for different values of $G_{r}$ Grashof number $\alpha$ Reynolds model viscosity parameter per axial distance $x$.

Figs.(13 -17) drawn for streamline patterns. The formation of circulating bolus of the fluid closed streamline is called trapping .this phenomenon is useful in grasping the motion of gastrointestinal tract and arrangement of thrombus in veins. The impacts of source/sink parameter, wavelength, Hartman parameter, viscosity parameter, are analyzed through these figures. It is important to observe that the size of trapping bolus reduces when the magnitude of said parameters $(\beta),(\lambda)(M),(\alpha)$, and $\phi$ increases. 


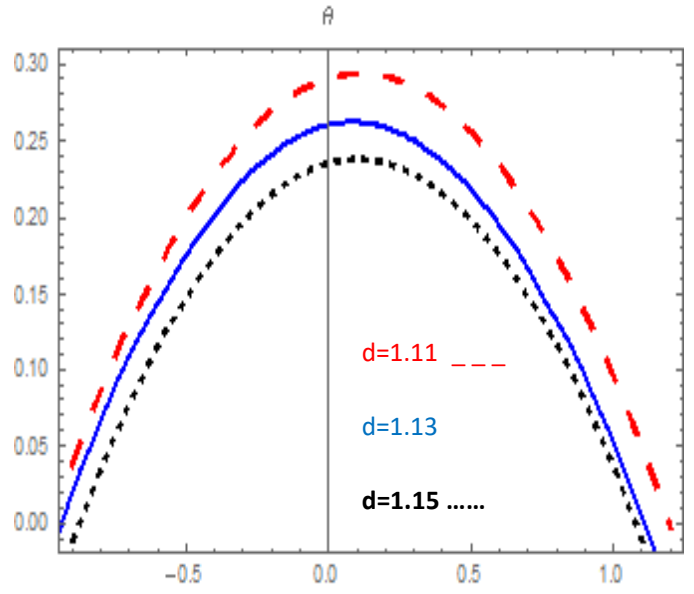

Fig.2.Temperature profile with different values of (d) values of $(\beta)$

$\mathrm{a}=\mathrm{b}=0.5, \beta=0.5, \phi=\frac{\pi}{4}, \mathrm{~m}=0.2, \mathrm{x}=0.5, \gamma=0.1$.

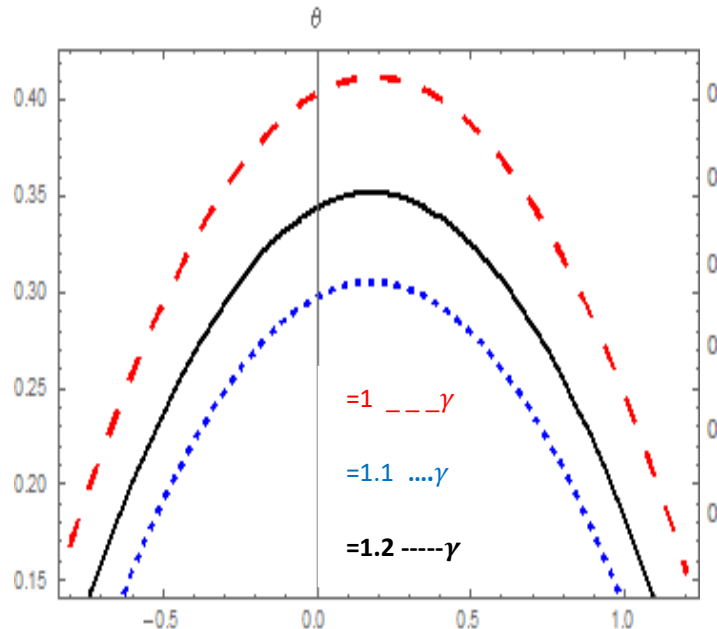

Fig.4.Temperature profile with different values of $(\gamma)$ values of $(\phi)$

$\mathrm{a}=\mathrm{b}=0.5, \mathrm{~d}=1, \phi=\frac{\pi}{4}, \mathrm{~m}=0.2, \mathrm{x}=0.5, \beta=0.5$.

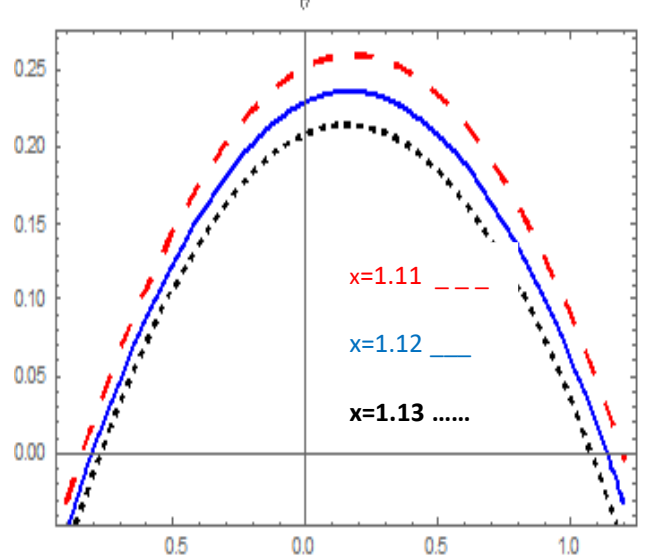

Fig.6.Temperature profile with different values of (x) values of (a)

$\mathrm{a}=\mathrm{b}=0.5, \mathrm{~d}=1, \phi=\frac{\pi}{4}, \mathrm{~m}=0.2, \gamma=0.1, \beta=0.5$. $=\frac{\pi}{4}$

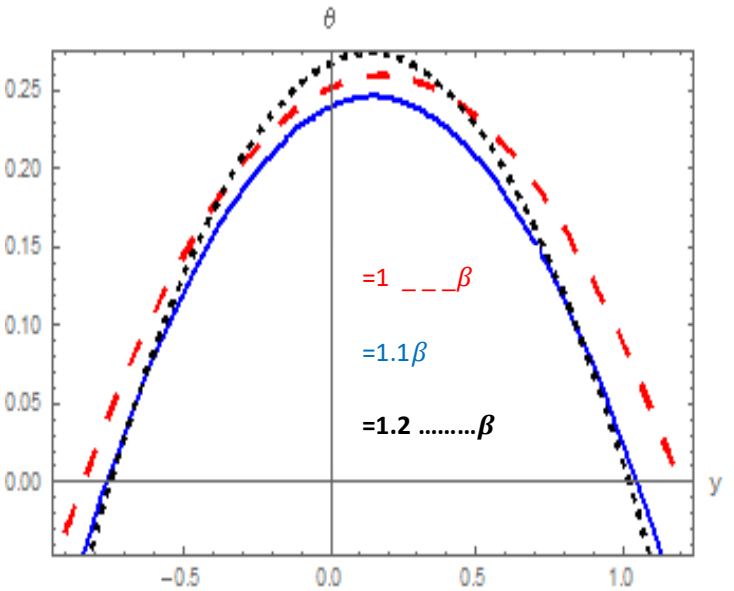

Fig.3. Temperature profile with different $\mathrm{a}=\mathrm{b}=0.5, \mathrm{~d}=1, \phi=\frac{\pi}{4}, \mathrm{~m}=0.2, \mathrm{x}=0.5, \gamma=0.1$.

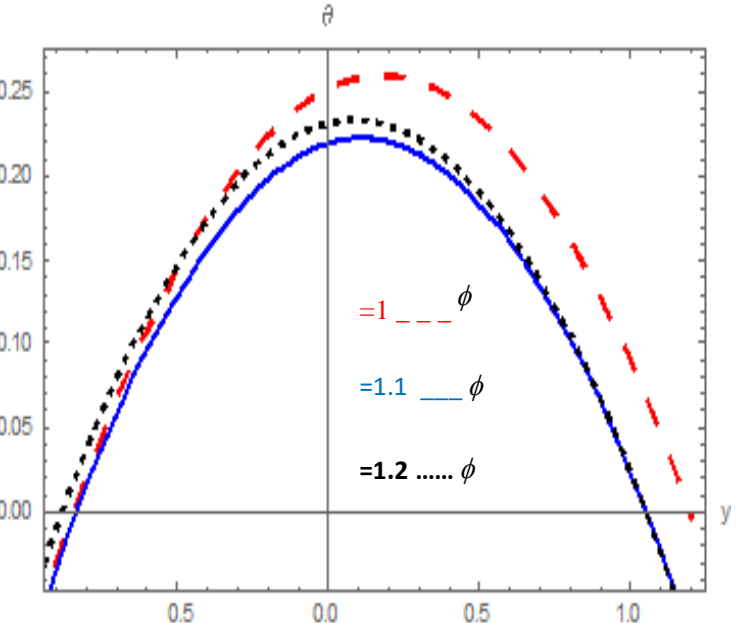

Fig.5. Temperature profile with different $\mathrm{a}=\mathrm{b}=0.5, \mathrm{~d}=1, \gamma=0.1, \mathrm{~m}=0.2, \mathrm{x}=0.5, \beta=0.5$

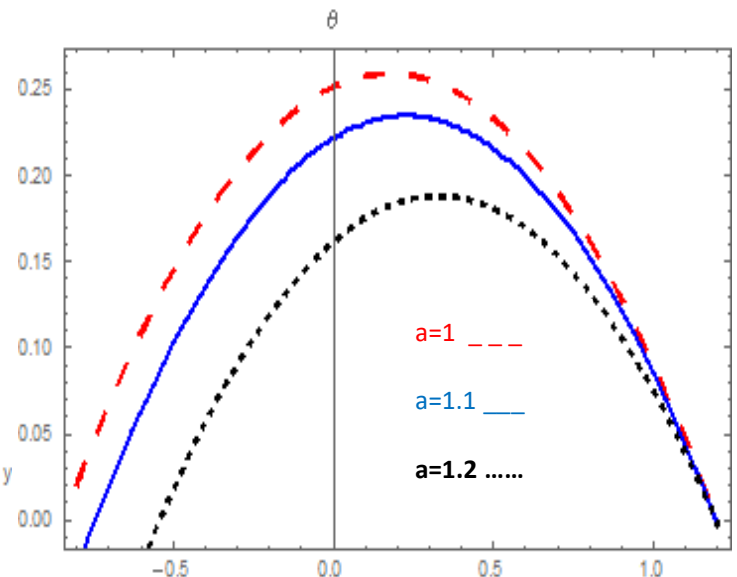

Fig.7. Temperature profile with different $\mathrm{b}=0.5, \mathrm{~d}=1, \gamma=0.1, \mathrm{~m}=0.2, \mathrm{x}=0.5, \beta=0.5, \phi$ 

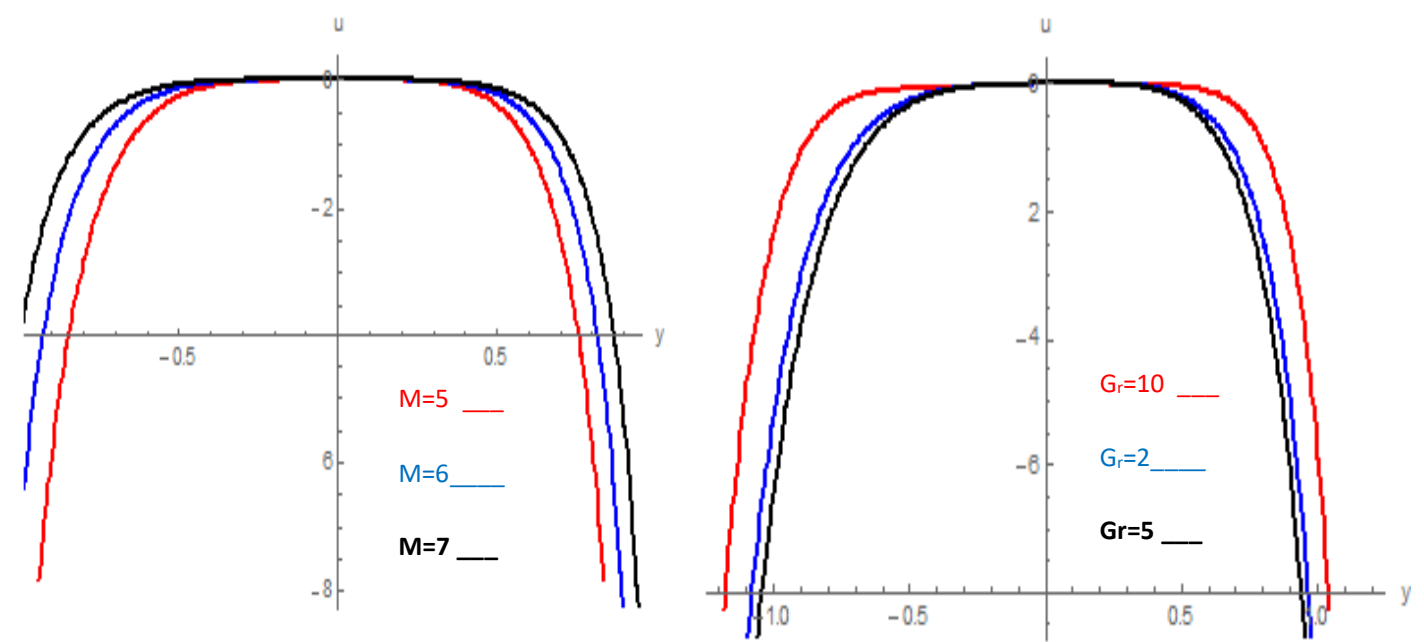

Fig.8. Velocity distribution with different values of (M)

Fig.9. Velocity distribution with different values of $\left(G_{r}\right)$

$\mathrm{a}=\mathrm{b}=0.5, \mathrm{~d}=1, \phi=\frac{\pi}{4}, \mathrm{~m}=0.1, \gamma=0.1, \beta=0.2, \mathrm{x}=0.1, \lambda=0.1$, $\mathrm{a}=\mathrm{b}=0.5, \mathrm{~d}=1, \phi=\frac{\pi}{4}, \mathrm{~m}=0.1, \gamma=0.1, \beta=0.2$, $\mathrm{x}=0.1, \lambda=0.1$, $\alpha=0.01, \mathrm{D}_{1}=4, \mathrm{~d}_{1}=0.2, \rho=1.057, \mathrm{G}_{\mathrm{r}}=2, \mathrm{~F}_{1}=0.6, \mathrm{~F}_{0}=0.8, \mu_{0}=0.4$. $\alpha=0.01, \mathrm{D}_{1}=4, \mathrm{~d}_{1}=0.2, \rho=1.057, \mathrm{M}=2, \mathrm{~F}_{1}=0.6, \mathrm{~F}_{0}=0.8, \mu_{0}=0.4$.

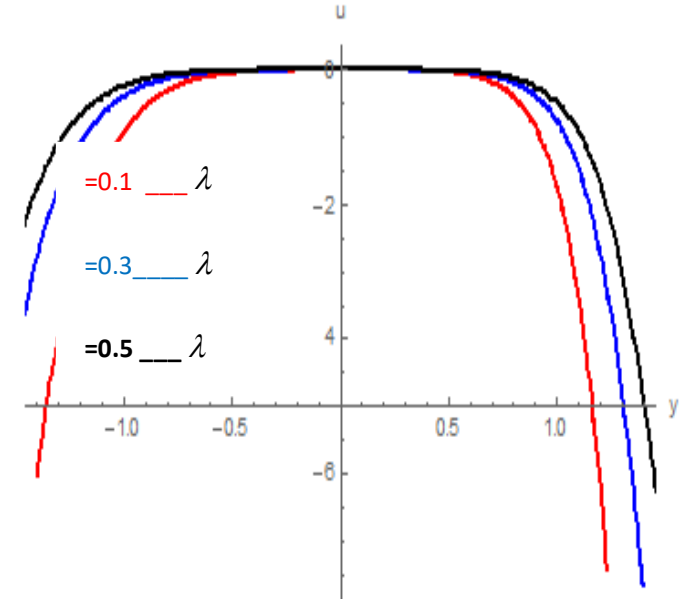

Fig.10. Velocity distribution with different values of $(\lambda)$ $\mathrm{a}=\mathrm{b}=0.5, \mathrm{~d}=1, \phi=\frac{\pi}{4}, \mathrm{~m}=0.1, \gamma=0.1, \beta=0.2, \mathrm{x}=0.1, \mathrm{M}=2$, $\alpha=0.01, \mathrm{D}_{1}=4, \mathrm{~d}_{1}=0.2, \rho=1.057, \mathrm{G}_{\mathrm{r}}=2, \mathrm{~F}_{1}=0.6, \mathrm{~F}_{0}=0.8, \mu_{0}=0.4$.

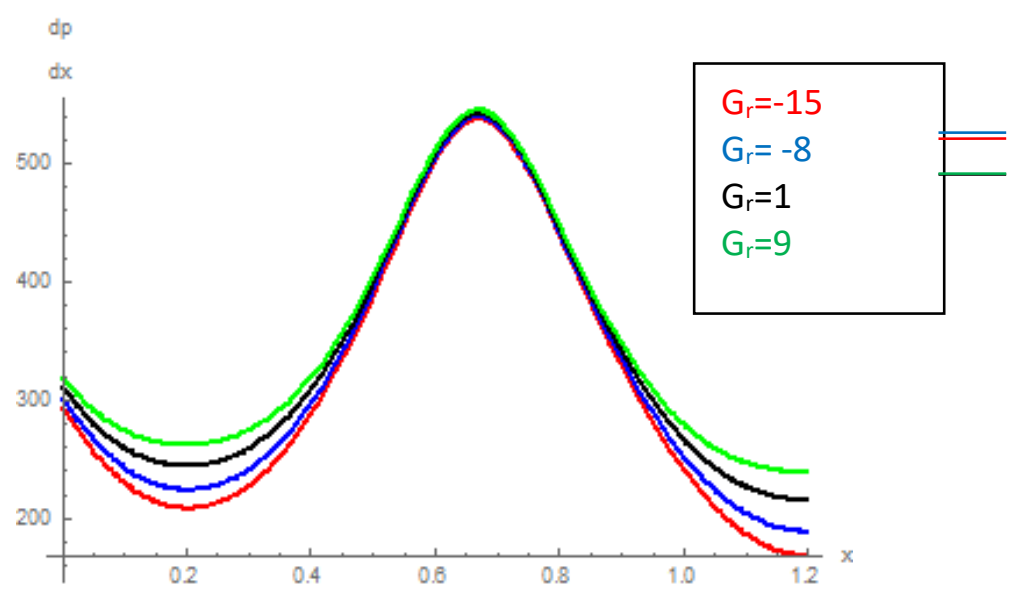


Fig 11.The pressure gradient $\frac{d p}{d x}$ vs. axial distance $(x)$ for different values of $G_{r}$ with Various instants $\mathrm{a}=\mathrm{b}=0.5, \mathrm{~d}=1, \mathrm{M}=2, \beta=0.2, \gamma=0.1, \alpha=0.01$. $\mathrm{F}_{0}=0.8, \mathrm{~F}_{1}=0.6, \mu_{0}=0.4, \mathrm{D}_{1}=4, \mathrm{~d}_{1}=0.2, \rho=1.057$.

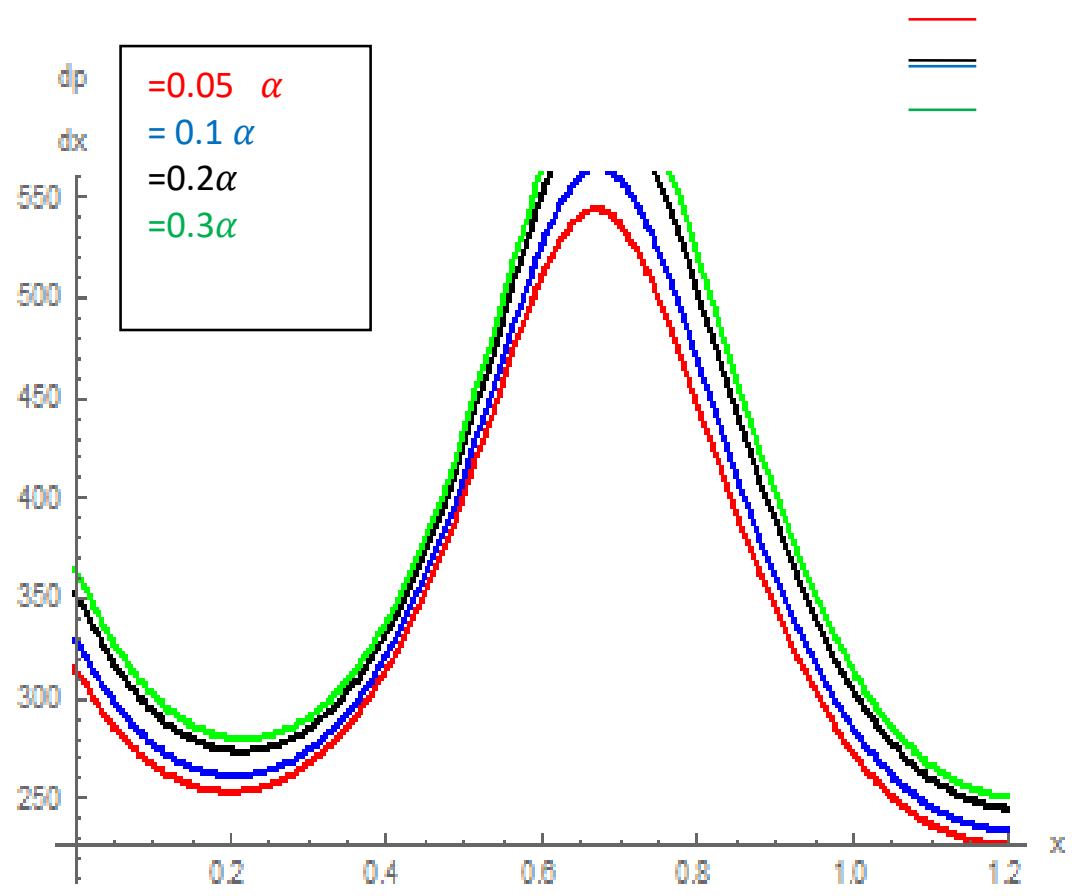

Fig 12.The pressure gradient $\frac{d p}{d x}$ vs. axial distance $(x)$ for different values of $\alpha$ with Various instants a=b =0.5,d=1,M=2, $\beta=0.2, \gamma=0.1, G_{r}=5$. $\mathrm{F}_{0}=0.8, \mathrm{~F}_{1}=0.6, \mu_{0}=0.4, \mathrm{D}_{1}=4, \mathrm{~d}_{1}=0.2, \rho=1.057$.

(a)

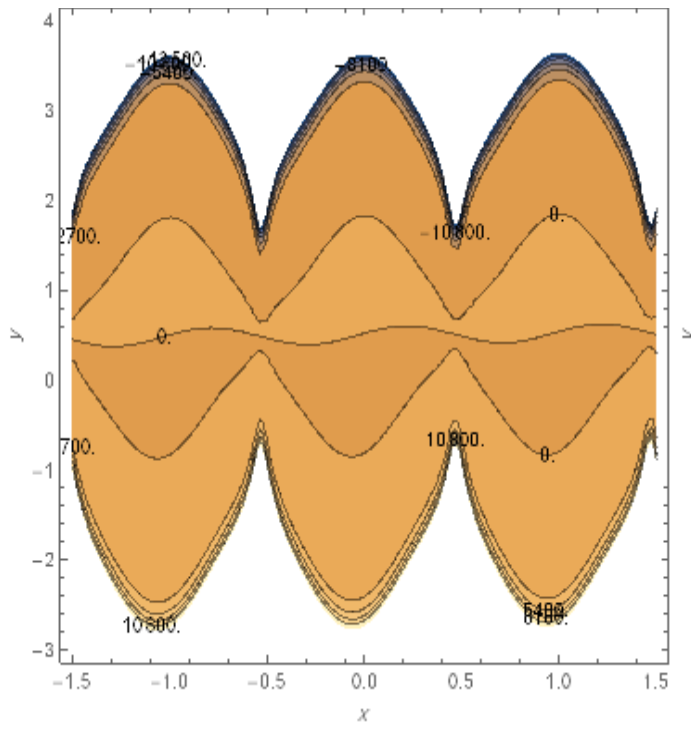

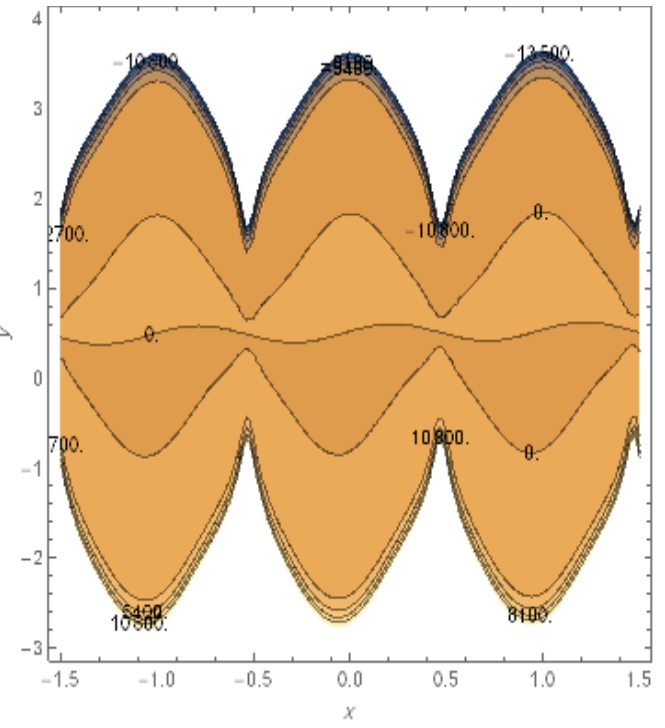

(c) (b) 


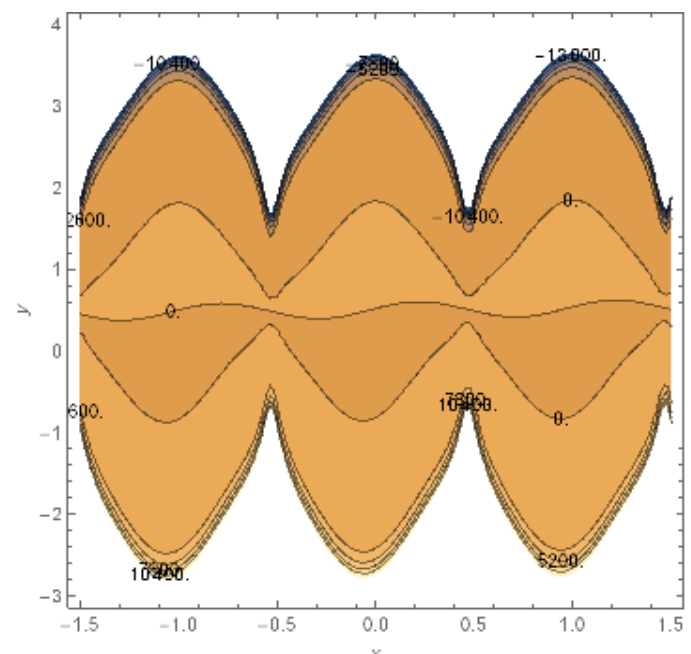

Fig 13.Stream line for different values of $\beta$.(a) $\beta=0.05$, (b) $\beta=0.2$, (c) $\beta=0.9$, and

Various parameters are $\mathrm{a}=\mathrm{b}=0.5, \mathrm{~d}=1, \mathrm{M}=2, \mathrm{~m}=0.2, \gamma=0.1, \Omega=0.4$.

$\mathrm{F}_{0}=0.5, \mathrm{~F}_{1}=0.1, \mu_{0}=0.4, \mathrm{D}_{1}=4, \mathrm{~d}_{1}=0.2, \rho=1.057, \phi=\frac{\pi}{4}, \alpha=0.05, \lambda=0.65$.

(a)
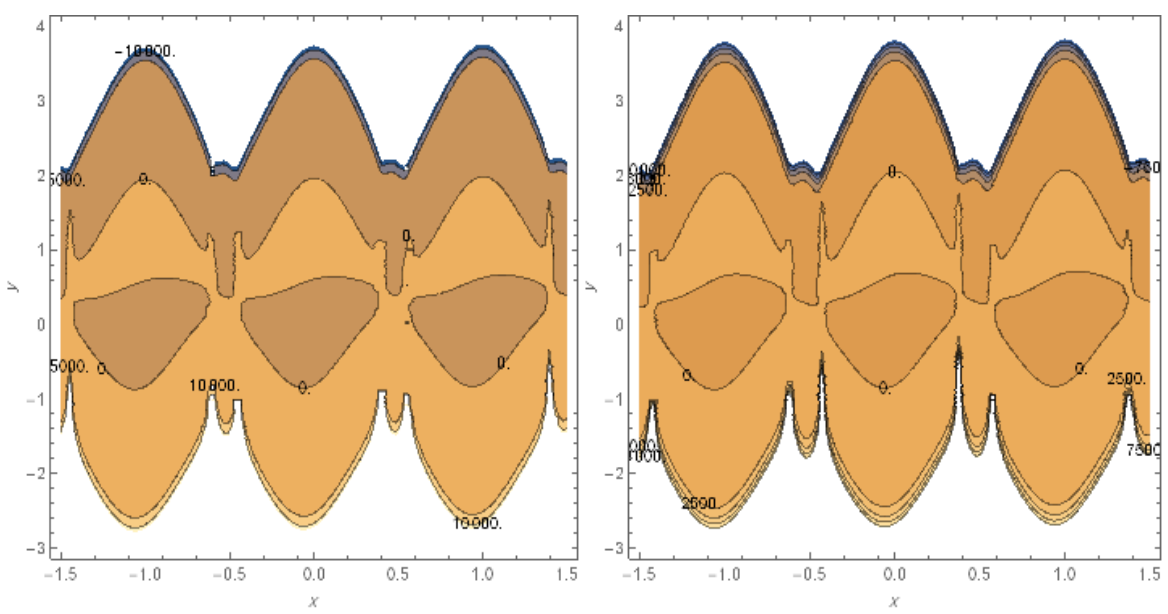

(b)

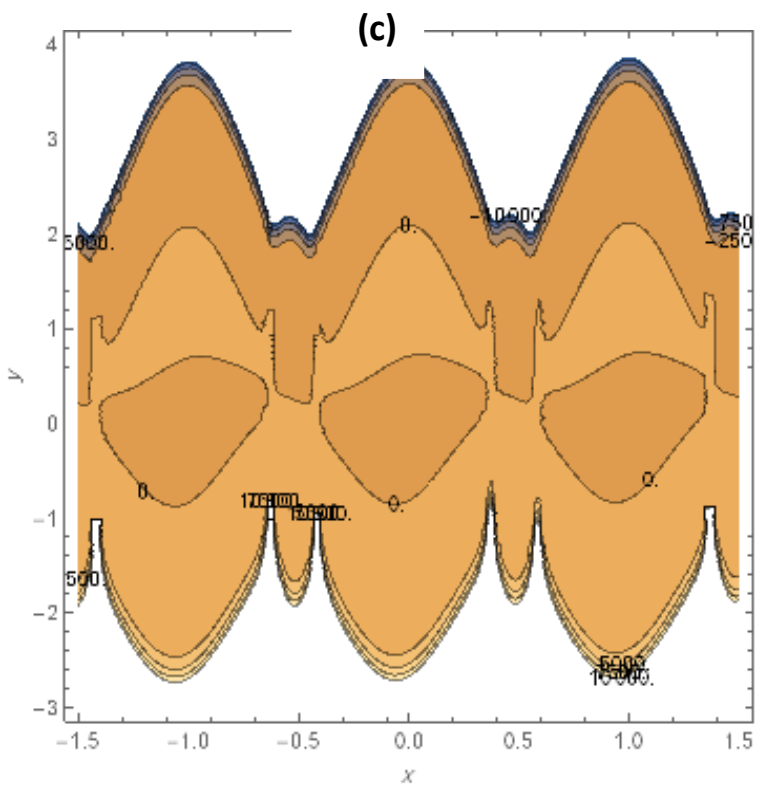

Fig 14.Stream line for different values of $\lambda$.(a) $\lambda=0.65$,(b) $\lambda=0.75$,(c) $\lambda=0.8$, and 
Various parameters are $\mathrm{a}=\mathrm{b}=0.5, \mathrm{~d}=1, \mathrm{M}=2, \mathrm{~m}=0.2, \gamma=0.1, \Omega=0.4$.

$\mathrm{F}_{0}=0.5, \mathrm{~F}_{1}=0.1, \mu_{0}=0.4, \mathrm{D}_{1}=4, \mathrm{~d}_{1}=0.2, \rho=1.057, \phi=\frac{\pi}{4}, \alpha=0.05, \beta=0.2$.

(a)
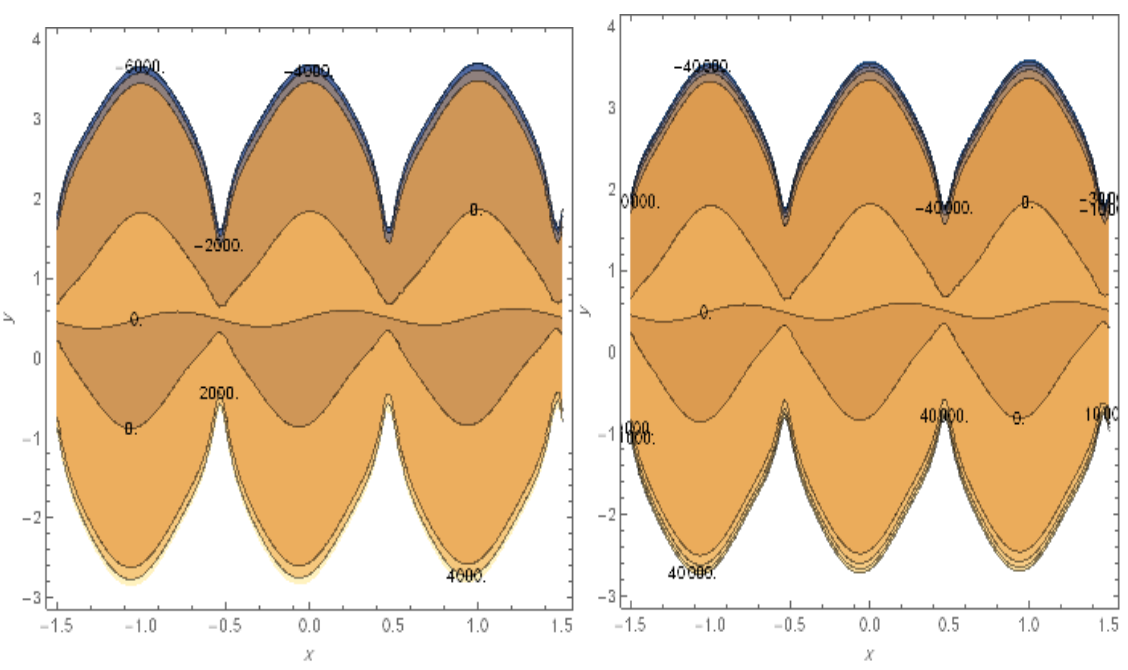

(b)

(c)

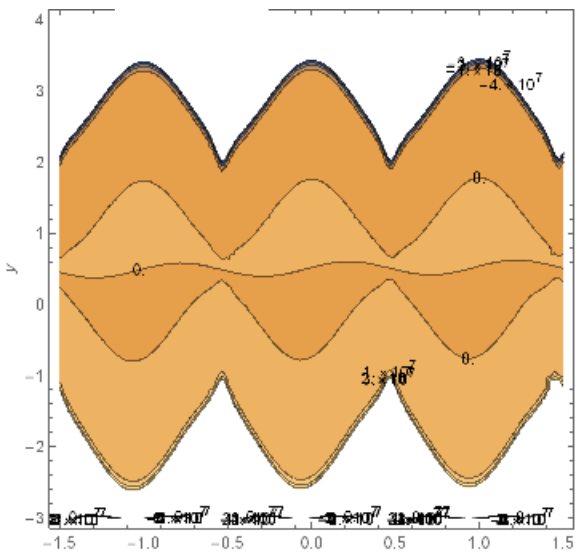

Fig 15.Stream line for different values of.(a) $\mathrm{M}=3$,(b) $\mathrm{M}=5$,(c) $\mathrm{M}=10$, and

Various parameters are $\mathrm{a}=\mathrm{b}=0.5, \mathrm{~d}=1, \lambda=0.5, \mathrm{~m}=0.2, \gamma=0.1, \Omega=0.5$.

$\mathrm{F}_{0}=0.5, \mathrm{~F}_{1}=0.1, \mu_{0}=0.4, \mathrm{D}_{1}=4, \mathrm{~d}_{1}=0.2, \rho=1.057, \phi=\frac{\pi}{4}, \alpha=0.05, \beta=0.2$.

(a)

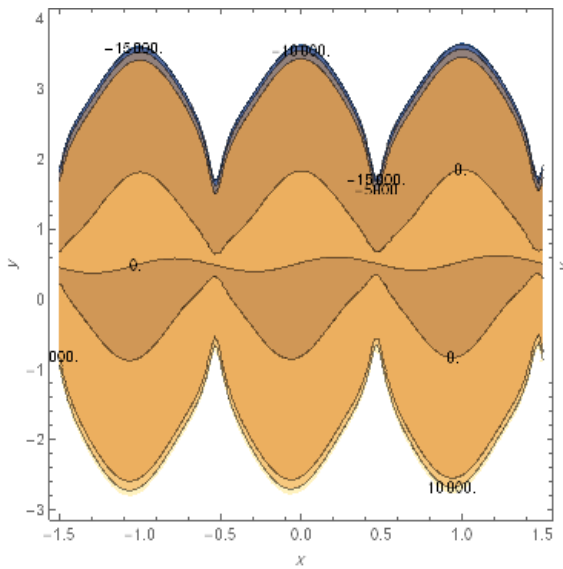

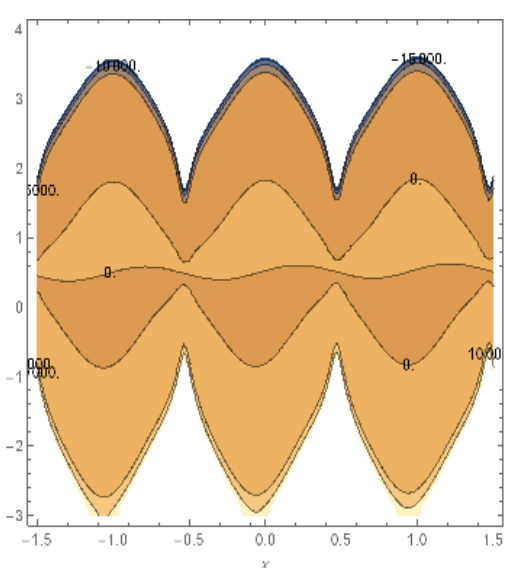

(c) 


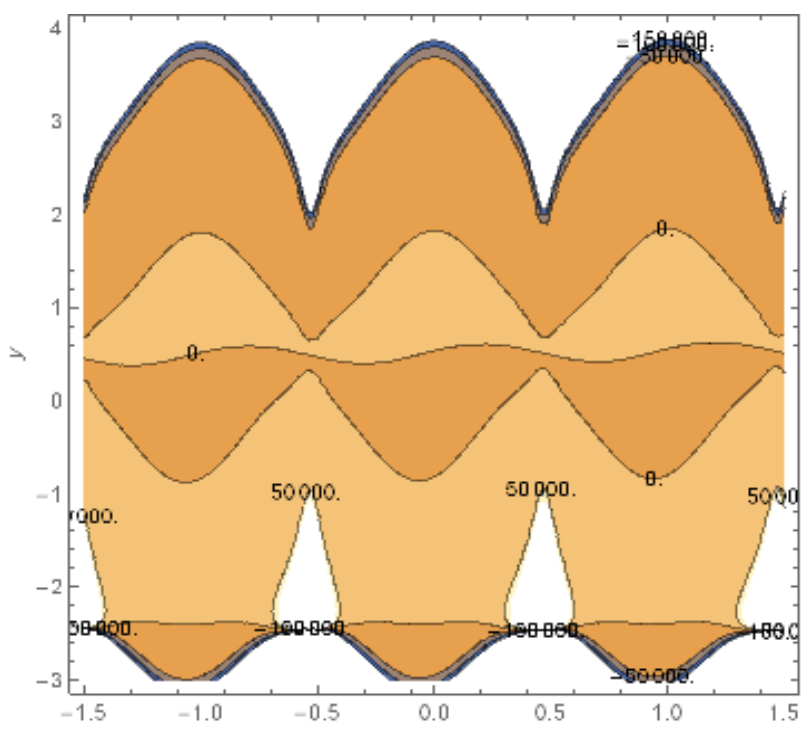

Fig 16.Stream line for different values of.(a) $\alpha=0.05$,(b) $\alpha=0.1$,(c) $\alpha=0.2$, and

Various parameters are $\mathrm{a}=\mathrm{b}=0.5, \mathrm{~d}=1, \lambda=0.5, \mathrm{~m}=0.2, \gamma=0.1, \Omega=0.5$.

$$
\mathrm{F}_{0}=0.5, \mathrm{~F}_{1}=0.1, \mu_{0}=0.4, \mathrm{D}_{1}=4, \mathrm{~d}_{1}=0.2, \rho=1.057, \phi=\frac{\pi}{4}, \mathrm{M}=4, \beta=0.2 \text {. }
$$

(a)
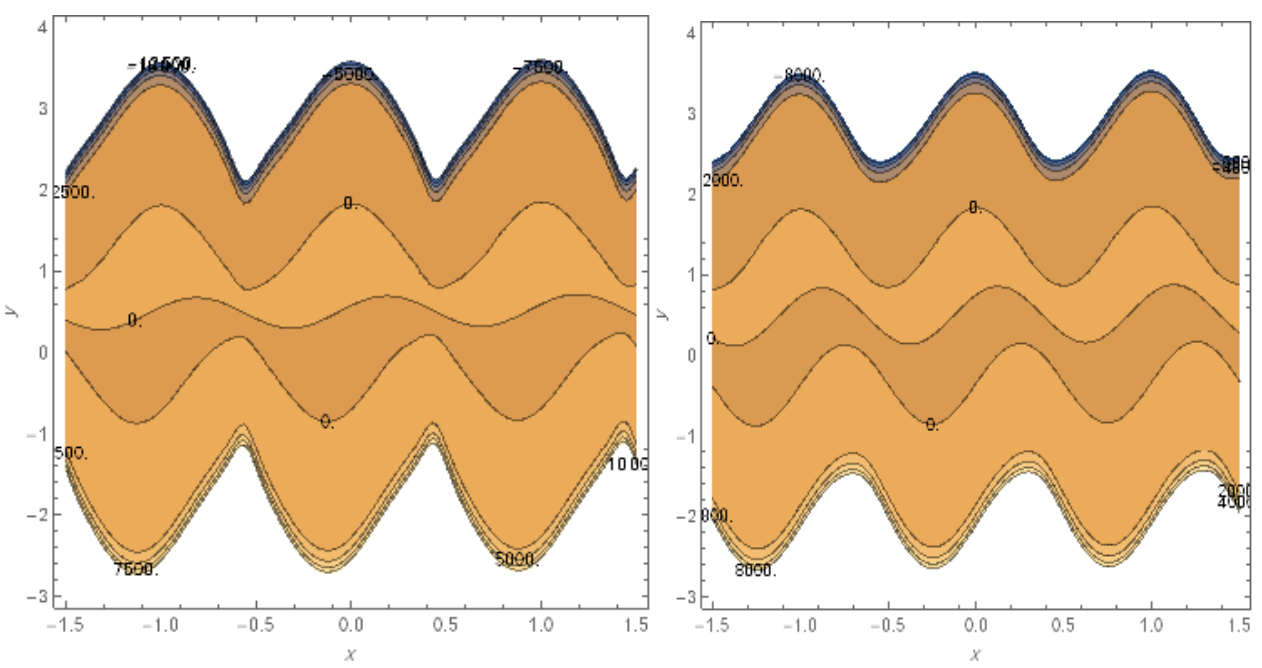

(c)

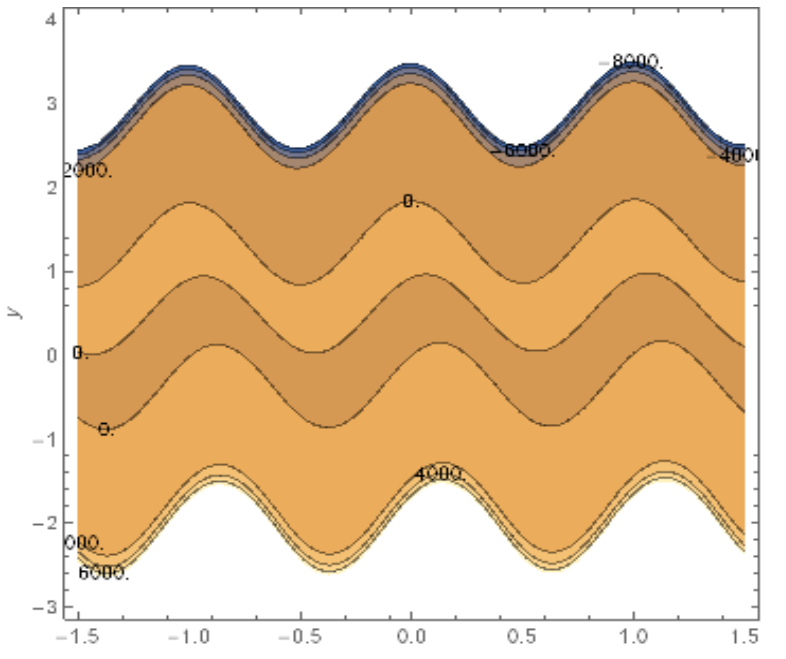

Fig 17.Stream line for different values of.(a) $\phi=\pi / 4$, (b) $\phi=\pi / 2$, (c) $\phi=3 \pi / 4$, and 


$$
\begin{aligned}
& \text { Various parameters are } \mathrm{a}=\mathrm{b}=0.5, \mathrm{~d}=1, \lambda=0.5, \mathrm{~m}=0.2, \gamma=0.1, \Omega=0.5 \text {. } \\
& \mathrm{F}_{0}=0.5, \mathrm{~F}_{1}=0.1, \mu_{0}=0.4, \mathrm{D}_{1}=4, \mathrm{~d}_{1}=0.2, \rho=1.057, \alpha=0.05, \mathrm{M}=4, \beta=0.2 \text {. }
\end{aligned}
$$

\section{Conclusions}

In this mathematical model, we explain the effects of rotation, variable viscosity and temperature on peristaltic flow in an asymmetric channel through porous medium. Analytic solution with closed form are constructed for axial velocity, temperature and stream function. The main results and concluding remarks of this investigation can be drawn :

- The temperature distribution is increasing function with enhancement of values $(\mathrm{d}),(\gamma),(\mathrm{x})$ of and decreasing with rising values of $(\phi),(a)$ and $(\beta)$.

- Increasing of magnetic field decreasing pumping of peristaltic size of the trapped bolus .

- $\quad\left(G_{r}\right),(\mathrm{M})$ and $(\lambda)$ parameters have different effects for velocity distribution .

\section{References}

The pressure gradient decreasing with increasing of the amplitude $(\alpha)$.

1. Hayat T., Ambreen Afsar, M.Khan, and S.Asghar. 2007.Peristaltic transport of a third order fluid under the effect of a magnetic field . Computers and Mathematics with Applications , 53: 1074-1087.

2. Alaa W. Saleh,Ahmed M. Abdulhadi .2016. Effects of Couple Stress and Porous Medium on Transient Magneto Peristaltic Flow under the Action of Heat Transfer. IOSR Journal of Mathematics , 12(4) : 71-83.

3. Abd El Naby, A., El Misery,A.E.M.and El Shamy I.I. 2004. Effects of an endoscope and fluid with variable viscosity on peristaltic motion, Applied Mathematics and Computation, 58(2):497-511 .

4. Hayat, T.and Ali, N.2008 . Effect of variable viscosity on the peristaltic transport of a Newtonian fluid in an asymmetric channel .Applied Math. Modell. $32: 761-774$.

5. Manjunatha S., Gireesha B.J.2016 . Effects of variable viscosity and thermal conductivity on MHD flow and heat transfer of a dusty fluid . Ain Shams Engineering Journal . $7: 505-515$.

6. E.F. Elshehawey, Z.M. Gharsseldien $\mathbf{. 2 0 0 4}$. Peritaltic transport of three - layered flow with variable viscosity, Appl.Math.Comput.153:417-432 .

7. Hayat T., Asghar Z., Asghar S., and Mesloub S . 2010. Influence of inclined magnetic field on peristaltic transport of fourth grade fluid in an inclined asymmetric channel .41(5): 553-563.

8. Mahmoud S.R., Abd-Alla A. M. and El-Sheikh M. A. 2011 . Effect of the rotation on wave motion through cylindrical bore in a micropolar porous medium .International Journal of Modern Physics B , 25(20):2713-2728

9. Siddiqui A.M., Schehawey W.H. 1994 .Peristaltic flow of second - order fluid in tubes ,Journal of Non-Newtonian Fluid Mech. , 53:257-284 .

10. Abd-Alla A. M., Yahya G. A., Mahmoud S.R. and Alosaimi H. S.2012. Effects of the rotation , magnetic field and initial stress on peristaltic motion of micropolar fluid, Meccanica ,47:1455-1465 .

11. Abd-Alla A. M., Abo-Dhahab S.M. ,Maram Albalawi , 2015. Effect of variable viscosity on periataltic flow of second order fluid with heat and mass transfer ,Journal of Comput. Theor .Nanosci ,12:3110-3117.

12. Abd-Alla A. M., Abo-Dhahab S.M. , 2015. Effect of an endoscope and rotation on the peristaltic flow involving a Jeffrey fluid with magnetic field, J . Braz . Soc. Eng. 37: 1277-1289 .

13. Abd-Alla A. M., Abo-Dhahab S.M. , Maram M. albalawi ,2015. Radially varying magnetic field on the peristaltic flow in a tube with an endoscope under the effect of rotation .J. Comput. Theor. Nanosci. 12:3066-3075.

14. Abd-Alla A. M., Abo-Dhahab S.M. ,2016.Rotation effect on peristaltic transport of a Jeffrey fluid in an asymmetric channel with gravity field ,Alexandria Engineering Journal .55:1725-1735.

15. Sheikholeslami M. , Abelman S. ,2015. Two-phase simulation of nanofluid flow and heat transfer in an annulus in the presence of an axial magnetic field .IEEE Trans. Nanotechnol.14:561-569.

16. Iftikhar, Naheeda, Abdul Rehman Hind Sadaf and Muhammad Najam Khan.2018.Impact of wall properties on the peristaltic flow of $\mathrm{Cu}$-water nano-unform inclined tube .International Journal of Heat and Mass Transfer 125:772-779.

17. Slattery, J.C. 1972 . Momentum Energy and Mass Transfer in Continua . McGraw Hill, New York . 